\title{
Tumor Heterogeneity in Endometrial Carcinoma: Practical Consequences
}

\author{
Sònia Gatius ${ }^{a, f}$ Dolors Cuevas ${ }^{a, f}$ Carlos Fernández ${ }^{a}$ Berta Roman-Canal ${ }^{a}$ \\ Virginia Adamoli ${ }^{a} \quad$ Josep Maria Piulats ${ }^{d} \quad$ Núria Eritja $^{a, f} \quad$ Mireia Martin-Satue ${ }^{c}$ \\ Gema Moreno-Bueno ${ }^{e, f}$ Xavier Matias-Guiua, b, f \\ ${ }^{a}$ Hospital Universitari Arnau de Vilanova, IRBLLEIDA, Lleida, ${ }^{\mathrm{b}}$ Hospital Universitari de Bellvitge, ${ }^{\mathrm{C}}$ Universitat de \\ Barcelona, and d Institut Català d'Oncologia, IDIBELL, L'Hospitalet de Llobregat, ${ }^{e}$ MD Anderson Cancer Center, \\ and ${ }^{\mathrm{f} C e n t r o ~ d e ~ I n v e s t i g a c i o ́ n ~ B i o m e ́ d i c a ~ e n ~ R e d ~ d e ~ O n c o l o g i ́ s, ~ M a d r i d, ~ S p a i n ~}$
}

\author{
Keywords \\ Endometrial carcinoma - Heterogeneity · Histologic \\ type - Endometrioid/serous tumors · Biomarker . \\ Immunohistochemistry
}

\begin{abstract}
Endometrial carcinoma (EC) shows intertumor heterogeneity, with several different histologic types differing in their morphologic and molecular features. There is also intratumoral morphologic heterogeneity, with different neoplastic cell components within the same tumor, with different morphologic and molecular features. In this article, we discuss the consequences of tumor heterogeneity in EC at the morphologic and molecular levels, by describing some illustrative examples produced by the research team. They are (1) morphologic heterogeneity in conventional EC and mixed tumors, (2) EC with microsatellite instability, (3) branched evolution as shown by exome sequencing analysis, (4) morphologic, molecular, and metabolomic differences between the tumor surface and myometrial invasion front, (5) tumor heterogeneity at the microenviromental level, (6) the sensitivity of endometrial aspirates to detect tumor heterogeneity in EC, and (7) sampling strategies to detect tumor hetero-
\end{abstract}

\section{KARGER}

(c) 2017 S. Karger AG, Basel

E-Mail karger@karger.com

www.karger.com/pat geneity in hysterectomy specimens. Tumor heterogeneity may have an important clinical impact, since it can be challenging to identify minor tumor cell populations that may have an impact on diagnosis, prognosis, and therapeutic decisions for patients with EC.

(c) 2017 S. Karger AG, Basel

\section{Introduction}

Endometrial carcinoma (EC) shows intertumor heterogeneity at the morphologic level, with different histologic types that have different morphologic features. The 2 most frequent types are endometrioid EC (EEC), and serous carcinoma (SC), which have different risk factors, prognosis, patterns of metastasis, and microscopic appearance $[1,2]$. There is also intratumoral morphologic heterogeneity. There is evidence that morphologic and molecular features are heterogeneously present in different tumor cell clones in a high proportion of ECs. There is a wide spectrum of heterogeneous tumors, ranging from EEC, with subtle variations in cytologic or architectural patterns, to mixed tumors, composed of 2 different histologic types (i.e., EEC and SC) in the same tumor. We 
discuss the consequences of tumor heterogeneity in EC at the morphologic and molecular levels, by describing some illustrative examples. Tumor heterogeneity may have an important clinical impact, since it can be a challenge to identify minor tumor cell populations that may have an impact on diagnosis, prognosis, and therapeutic decisions. It is thus important to provide rules for pathologic sampling to ensure that these minor cell subpopulations are identified. We also assess tumor heterogeneity in a small series of EC, by comparing conventional sampling ( 1 block $/ 1-2 \mathrm{~cm}$ ) and complete sampling of the tumor, to estimate the possibilities of missing prognostically relevant cell clones with current conventional sampling protocols.

\section{Morphologic Heterogeneity in EC}

There are different histologic types of EC with different morphologic features $[1,2]$, the most characteristic being EEC and SC. EEC occurs in perimenopausal patients, in the setting of an excess of estrogen, and is preceded by precursor lesions (endometrial hyperplasia/endometrioid intraepithelial neoplasia). Under the microscope, EEC shows tubular glands of marked complexity, with fusion of the glands and cribriform masses but somewhat resembling the proliferative endometrium [3]. In contrast, $\mathrm{SC}$ occurs in postmenopausal patients in the absence of hyperestrogenism. Microscopically, SC has thick, fibrotic or edematous papillae with a prominent stratification of tumor cells, cellular budding, and anaplastic cells with large, eosinophilic cytoplasms [4]. EEC tends to be restricted to the uterus with occasional extension to the retroperitoneal lymph nodes, while SC has a strong tendency for lymph node and peritoneal metastasis. The molecular alterations involved in the development of EEC are different from those of SC. EEC shows microsatellite instability (MI) as well as mutations in $P T E N, K-R A S$, and CTNNB1 whereas SC exhibits alterations of p53, a widespread loss of heterozygosity (reflected by chromosomal instability) as well as other molecular alterations (STK15, p16, E-cadherin, and C-erbB2). PIK3CA and ARID1A mutations have been identified in both tumor types. The Cancer Genome Atlas Research Network (TCGA) has recently performed an integrating genomic characterization of EC. The genes most frequently mutated in EEC were PTEN (77\%), PIK3CA (53\%), PIK3R1 (37\%), CTNNB1 (36\%), ARID1A (35\%), K-RAS (24\%), CTCF (20\%), RPL22 (12\%), TP53 (11\%), FGFR2 (11\%), and ARID5B (11\%). The genes most fre- quently mutated in SC were TP53 (90.7\%), PIK3CA (41.9\%), FBXW7 (30.2\%), PPP2R1A (36.6\%), CHD4 (16.3\%), CSMD3 (11.6\%), and COLA 11 (11.6\%) [5].

Morphologic intratumor heterogeneity is frequently seen in otherwise conventional EEC, in which different cytologic (columnar, mucinous, or squamous) or architectural (glandular, papillary, or solid) features may coexist in the same tumor. Coexistence of glandular/papillary morphology with a solid pattern is particularly relevant since histologic grading (an important parameter to predict prognosis) is dependent on the percentage of solid areas. Intratumor heterogeneity at the architectural level explains histologic discrepancies between the initial biopsy and the hysterectomy specimen. This is important because misinterpretation of the histologic grade in the biopsy may lead to an inappropriate surgical strategy. Moreover, tumors with focal solid (high-grade) areas are sometimes heterogeneous for p53 alterations (Fig. 1).

However, the typical examples of tumor heterogeneity at the morphologic level are mixed tumors, composed of $>1$ different histologic type. There are 3 types of mixed EC: (1) mixed EEC-SC, (2) dedifferentiated carcinomas, i.e., undifferentiated carcinomas (UC) coexisting with low-grade EEC, and (3) malignant mixed Müllerian tumors (MMMT) (having a biphasic pattern, with epithelial malignant elements and a sarcomatoid component).

Regarding mixed EEC-SC, it has been suggested that the SC component originates as a result of tumor progression from a preexisting EEC, since frequently, these tumors retain the molecular alterations of typical EEC. In a collaborative study, our group studied, for the first time in the literature, the mutation profiles of the separate tumor components in 23 mixed EEC-SC, using the MassARRAY compact analyzer, in comparison with pure EEC and pure SC. We observed that, in 6 out of 23 (26.1\%) mixed EEC-SC, all somatic mutations were shared by the different components. In contrast, in 2 out of 23 (13.0\%) mixed EEC-SC, mutations were only found in the EEC component but in 6 tumors, different mutations were found in both components. The frequency of the K-RAS and PTEN mutations in the EEC component of mixed EEC-SC (21.7 and 13.0\%, respectively) was similar to that in pure EEC (17.0 and 19.6\%), and higher than in pure SC (12.5 and 0\%). Moreover, the frequency of TP53 mutations in the SC component of mixed EEC-SC (17.4\%) was similar to that in pure SC (16.7\%), and significantly higher than that in pure EEC (1.7\%). Interestingly, the SC component of mixed EEC-SC had frequent mutations in $K-R A S$ and PTEN (17.4 and 8.7\%), while the EEC component of mixed EEC-SC had frequent mutations in TP53
36

Pathobiology 2018;85:35-40

DOI: $10.1159 / 000475529$
Gatius et al. 
Fig. 1. Heterogeneous distribution of $\mathrm{p} 53$ alteration. A p53 mutation was detected in $15 \%$ of tumor cells by next-generation sequencing, and correlated with focal positivity (mutated pattern) of p53 on immunohistochemistry.
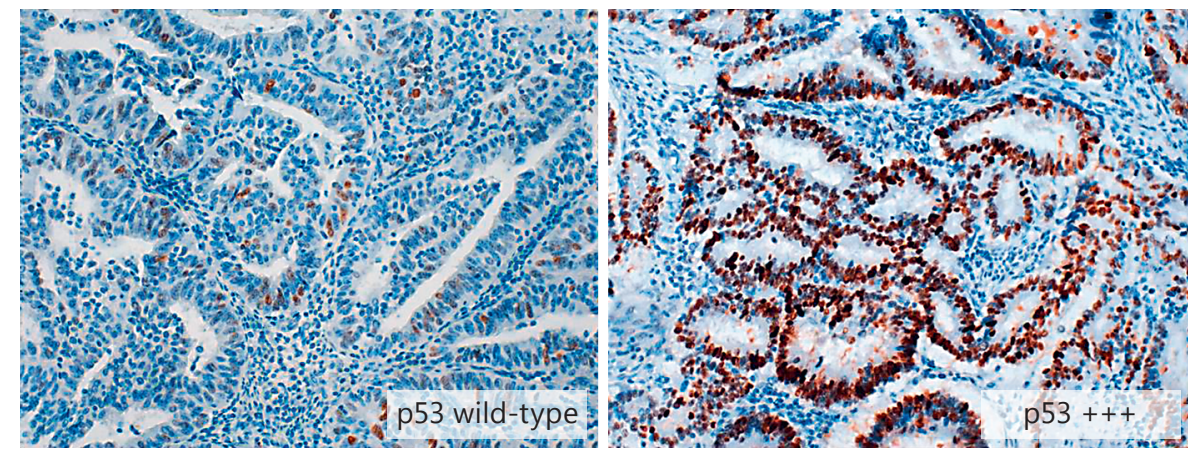

(13.0\%) [6]. The results show that the EEC and SC components of mixed EEC-SC show a molecular resemblance to pure EEC and SC. The results also suggest that mixed EEC-SC are heterogeneous tumors, with 2 different components that result from divergent tumor progression. The percentage for the minor component has been arbitrarily established at $5 \%$, but it is usually recommended that any amount of SC is reported because of the adverse prognostic value.

It is generally accepted that in dedifferentiated carcinoma, the UC component arises as a consequence of tumor progression from the preexisting EEC elements. Several groups of investigators have reported that (MI) is a frequent molecular genetic alteration in these tumors. A role for CTNNB1, PPP2R1A, and TP53 as drivers of progression from EEC to UC has been suggested. However, our data suggest different progression pathways that depend on the molecular subtype of the EEC component. Thus, EEC with a mismatch repair deficiency progress to UC by accumulating mutations in the genes that are regularly altered in EC, such as ARIDIA, PIK3CA, and CTNNB1. However, microsatellite-stable EEC with the PTEN mutation progress to UC, by TP53 mutations. Finally, POLE-mutated EEC progress to UC by accumulating mutations in the genes characteristic of EEC and/or TP53 mutations [7].

MMMT, like sarcomatoid carcinomas of other locations, probably develop via the epithelial-mesenchymal transition (EMT) in EC. Whereas the transient occurrence of the EMT phenomenon is important for myometrial invasion in conventional EC, MMMT show permanent expression of EMT, leading to the repression of epithelial markers and the increased expression of mesenchymal markers including the proteins involved in skeletal muscle development. MMMT are heterogeneous by definition, because of their characteristic biphasic pattern. However, in some cases, it is clear that MMT also arise from preexisting SC (or, less frequently, EEC), since the 2 tumor components are present in the uterine mass. In such cases, the tumor heterogeneity is more obvious. Identification of the MMT component is important because of the adverse prognosis associated with this particular type of tumor [8].

\section{EC with MI Are Composed of Heterogeneous Clones with Different Genetic Alterations}

An EEC with MI provides a good scenario to assess the molecular features associated with tumor heterogeneity. The MI-associated mismatch repair deficiency leads to the accumulation of myriads of mutations in the coding and noncoding DNA sequences. Short-tandem repeats, like microsatellites, are particularly susceptible to mismatch repair alterations, and are sometimes located within the coding sequence of some important genes: $B A X$, IGFIIR, hMSH3, hMSH6, MBD4, CHK-1, and Caspase-5. These genes may be potential targets in the process of the progression of MI-positive EC. Our group has demonstrated the heterogeneous presence of these secondary mutations in different regions of MI-positive EC as well their differential presence in lymph node metastases, suggesting that some of them are predominantly present in the tumor clones prone to developing metastasis $[9,10]$.

\section{Next-Generation Sequencing Confirms that EC Follows Branched Evolution}

A few studies have evaluated tumor heterogeneity in EC by exome sequencing. In a recent study, we assessed the mutational profile of synchronous endometrioid carcinomas of the endometrium and the ovaries by this method. Interestingly, results suggest that the vast major- 
ity of ovarian tumors that occur simultaneously with EEC are, indeed, a metastasis of the uterine tumor, although they are usually associated with a favorable prognosis [11, $12]$. Interestingly, we were able to perform exome sequencing analysis in the 3 different areas of the primary tumor and in 1 area of the ovarian mass in 1 case. Our results confirmed a pattern of branched evolution with a significant number of ubiquitous mutations, but also private mutations from different areas of the primary tumor and the ovarian metastasis [13].

\section{EC Show Different Proteomic and Metabolomic Features on the Surface and in the Invasive Area}

Intratumor heterogeneity is also seen when comparing tumor cells at the invasive front with the cells that are located on the surface of the tumor. Our group recently compared samples from the surface area and the myoinvasive front of EC in order to investigate whether the EMT program was involved [14]. We found increases in SLUG, ZEB1, and HMGA2 mRNA expression at the myoinvasive front of tumor samples, indicating the role of these transcriptional factors in EC progression and invasion. An increase in Snail and Twist expression occurred concomitantly with a decrease in E-cadherin expression at the myoinvasive front of early-stage EC. For a better understanding of the potential role of EMT in the genesis and development of ECC, an in vitro scenario mimicking this process was developed using IK V600E-transformed EC cells. The overexpression of the BRAF missense mutation V600E leads to a persistent activation of ERK1/2 and an increase in Snail protein levels, as demonstrated by immunofluorescence and Western blot analysis.

Furthermore, our group used a proteomic approach to characterize the specific components of the invasive front. Some of these proteins have already been described as specific to the invasive tumor front, like Fascin 1 in colorectal cancer, with a transient upregulation that promotes the acquisition of the migratory and invasive phenotypes that lead to metastasis. A more interesting result was the identification of different enzymes involved in oxidative stress like SOD1 or BLVRB. In addition to genetic alterations, the development and progression of malignant tumors have been related to cellular metabolism influences [15].

Our group has also assessed that the metabolomic profile of tumor cells at the invasive front differs from that on the tumor surface [16]. Using a nontargeted approach and focusing on the metabolite profiles of low-molecular- weight $(\mathrm{m} / \mathrm{z}<3,000)$ ionizable molecules, we detected 2,777 molecules. Multivariate analyses confirmed that changes in the metabolomic profile were associated with myometrial invasion. We performed the paired Student $t$ test and found that 135 molecules were statistically different on the surface and at the myometrial invasion front. We identified 11 molecules; of these, 7 had increased (xanthine, lactamide, $\alpha$-D-fucose, 3-mercaptopyruvate, ribitol, phosphocholine [32:0] and eicosapentaenoic acid) and 5 had decreased (inosine, deoxycytidine, hypoxanthine, CDP-ethanolamine, and $5^{\prime}$-methylthioadenosine) at the myometrial invasion front compared to on the surface.

Moreover, EEC may exhibit peculiar patterns of myometrial invasion, which may coexist in particular tumor types, leading to tumor heterogeneity at the invasive front. EEC with diffuse infiltration are not associated with a stromal response, while expansile-type invasion shows collective cell invasion. Our group is currently studying the expression of proteins related to the exocyst in this particular type of invasion, and we have demonstrated a role for cytoplasmic expression of cyclin D1 in several specific types of myometrial invasion. The localization of cyclin D1 in the cytoplasm and membranes was significantly higher in peripheral cells than in inner cells in collective and pushing invasion patterns. It was also high when tumors infiltrated as single cells, small clusters of cells, or in the so-called MELF pattern of invasion. We built a variant of cyclin D1 (Ccnd1-CAAX), that remains attached to the cell membrane, sequestering this cyclin in the cytoplasm and transfecting this variant to the EEC cell lines. The tumor cells harboring Ccnd1-CAAX showed high levels of invasiveness and metastatic potential compared with the wild-type allele of Cyclin D1, confirming a role for cytoplasmic cyclin Dlin invasion [17].

\section{The Heterogeneous Microenvironment in EC}

The microenvironment has been shown to play an important role in tumor progression. The tumor and the surrounding microenvironment (blood vessels, immune cells, fibroblasts, macrophages, and extracellular matrix) are closely related and interact constantly, through a wide range of growth factors, cytokines, and chemokines. Tumor microenvironment may also contribute to tumor heterogeneity, and has been shown to differ in its role in different tumor types, showing significant prognostic value, and even playing a role in resistance to treatment. The stroma is usually desmoplastic and contains inflammatory cells including foam macrophages, fibroblasts, and lym-
38

Pathobiology 2018;85:35-40

DOI: $10.1159 / 000475529$
Gatius et al. 
phocytes, but it may also vary in different areas of the tumor. In a recent study, different stromal signatures (a macrophage response associated with CSF1 and a desmoidtype fibroblastic response) were seen in EC. Interestingly, $13 \%$ of the EC showed the 2 stromal signatures in different areas, suggesting EC heterogeneity with regard to the composition of the tumor microenvironment [18].

\section{Aspirates Capture the Intratumor Heterogeneity of EC}

The diagnosis of EC is usually performed by endometrial biopsy, in which a small fragment of tumor tissue is obtained by endometrial aspiration. This is a good diagnostic method with good sensitivity and specificity for the diagnosis of cancer. However, in heterogeneous tumors, there is the risk that the biopsy does not contain the minor cell population that can have an impact on diagnosis, prognosis, and treatment decisions.

In a recent study, genetic sequencing analysis was performed on different tumor regions from 21 of the EC hysterectomy specimens [19]. A comparative mutation analysis revealed differences in the mutational profiles of the distinct regions of the EEC in 10/14 patients (71.4\%), confirming the presence of intratumor heterogeneity. A lower proportion of heterogeneity at the mutational level was seen in SC, and carcinosarcoma at the mutational level was attributed to the chromosomal instability that is frequently found in these aggressive tumor types. A comparative mutation analysis was performed on the different tumor regions of hysterectomy specimens and the tumor fragments that had been previously obtained in the initial aspiration biopsy. Interestingly, the results show that endometrial aspirates captured the heterogeneity of the tumor, probably because endometrial aspirates contain tumor tissue fragments from different areas that line the endometrial cavity. This is a very different situation from organs like the breasts, lungs, kidneys, and colon, in which the diagnostic biopsy is obtained by endoscopic or fine-needle biopsy from a specific part of the tumor, with a high risk of missing the minor subpopulation of tumor cells with morphologic and molecular features that are relevant for diagnosis and prognosis. The study described included several cases that showed discrepancy in histologic grade between the endometrial aspirate and the hysterectomy specimen, because of the different proportion of solid areas in the 2 samples. Interestingly, in the mutational analysis, we found strong agreement between the 2 samples, regardless of the proportion of solid areas.

\section{Conventional Sampling Is an Appropriate Approach to Capture Tumor Heterogeneity in Hysterectomy Specimens}

We also performed a pilot study. We assessed tumor heterogeneity in a small series of $16 \mathrm{EC}$ (13 EEC, 2SC, and $1 \mathrm{MMMT}$ ), by comparing conventional sampling ( 1 block per 1-2 cm) and complete sampling of the tumor, to estimate the possibilities of missing the prognostically relevant cell clones with current conventional sampling protocols. Several prognostic parameters were assessed in each of the 16 cases separately by analyzing the conventional and complete sampling. These included the stage, level of myometrial invasion, and presence of lymphovascular space invasion (with H\&E and CD31). The conventional approach was sufficient to appropriately assess these prognostic parameters, and the additional blocks did not add any significant information. We also compared the results obtained with the initial sampling and those obtained with the additional blocks, and the statistical correlations were assessed by means of the MannWhitney nonparametric test. There were no differences regarding stage, and no significant differences were seen when assessing myometrial invasion $(p=0.99)$, or lymphovascular space invasion $(p=0.61)$. In conclusion, our pilot study shows that a conventional approach based on the sampling of 1 block per $1-2 \mathrm{~cm}$ after appropriate gross inspection seems to be appropriate for the assessment of some of the prognostic parameters in EC.

\section{Summary}

There are many examples of intratumor heterogeneity in EC, either at the morphologic or the molecular level. Attention should be paid so as not to miss minor subpopulations of tumor cells with diagnostic and prognostic relevance. Interestingly, endometrial aspirates are able to capture the molecular heterogeneity of EC, probably because they contain cells from different areas of the tumor. Finally, a conventional sampling approach, with 1 tissue block/1-2 cm, seems to be enough for the detection of pathologic parameters (levels of myometrial invasion and lymphovascular space invasion) in a hysterectomy specimen of EC.

\section{Disclosure Statement}

The authors declare that there are no conflicts of interest. 


\section{References}

1 Yeramian A, Moreno G, Dolcet X, Catasus Ll, Abal M, Colás E, Reventós J, Palacios J, Prat J, Matías-Guiu X: Endometrial carcinoma: molecular alterations involved in tumor development and progression. Oncogene 2013;32: 403-413.

2 Matias-Guiu X, Prat J: Molecular pathology of endometrial carcinoma. Histopathology 2013;62:111-123.

3 Azueta A, Gatius S, Matias-Guiu X: Endometrioid carcinoma of the endometrium. Pathologic and molecular features. Sem Diagn Pathol 2010;27:226-240.

4 Gatius S, Matias-Guiu X: Practical issues in the diagnosis of serous carcinoma of the endometrium. Mod Pathol 2016;29(suppl 1): S45-S58.

5 Cancer Genome Atlas Research Network: Integrated genomic characterization of endometrial carcinoma. Nature 2013;497:67-73.

6 Coenegrachts L, Garcia-Dios DA, Depreeuw J, Santacana M, Gatius S, Zikan M, Moerman P, Verbist L, Lambrechts D, Matias-Guiu X Amant F: Mutation profile and clinical outcome of mixed endometrioid-serous endometrial carcinomas is different from pure endometrioid or serous carcinomas. Virchows Archiv 2015;466:415-422.

7 Rosa-Rosa JM, Leskelä S, Cristóbal-Lana E, Santón A, LópezGarcía MA, Muñoz G, PérezMies B, Biscuola M, Prat J, Oliva E, Soslow RA, Matias-Guiu X, Palacios J: Molecular genetic heterogeneity in undifferentiated endometrial carcinomas. Mod Pathol 2016;29: 1390-1398.

8 Castilla MÁ, Moreno-Bueno G, RomeroPérez L, De Vijver KV, Biscuola M, LópezGarcía MA, Prat J, Matías-Guiu X, Cano A, Oliva E, Palacios J: Micro-RNA signature of the epithelial-mesenchymal transition in endometrial carcinosarcoma. J Pathol 2011;223: 72-80.
9 Catasus Ll, Matias-Guiu X, Machín P, Muñoz J, Prat J: BAX somatic frameshift mutations in endometrioid adenocarcinomas of the endometrium: evidence for a tumor progression role in endometrial carcinomas with microsatellite instability. Lab Invest 1998;78:14391444.

10 Catasus Ll, Matias-Guiu X, Machin P, Zannoni GF, Scambia G, Benedetti Panici P, Prat $\mathrm{J}$ : Frameshift mutations at coding mononucleotide repeat microsatellites in endometrial carcinomas with microsatellite instability. Cancer 2000;7:132-139.

11 Prat J, Matias Guiu X, Barreto J: Simultaneous carcinoma involving the endometrium and the ovaries. A clinicopathologic, immunohistochemical and DNA cytometric study of 18 cases. Cancer 1991;68:2455-2459.

12 Irving JA, Catasús L, Gallardo A, Bussaglia E, Romero M, Matias-Guiu X, Prat J: Synchronous endometrioid carcinomas of the uterine corpus and ovary: alterations in the $\beta$-catenin (CTNNB1) pathway are associated with independent primary tumors and favorable prognosis. Hum Pathol 2005;36: 605-619.

13 Schultheis AM, Ng CK, De Filippo MR, Piscuoglio S, Macedo GS, Gatius S, Perez Mies B, Soslow RA, Viale A, Huberman KH, Palacios JC, Reis-Filho JS, Matias-Guiu X, Weigelt B: Massively parallel sequencing-based clonality analysis of synchronous endometrioid endometrial and ovarian carcinomas. J Natl Cancer Inst 2016;108:djv427.
14 Montserrat N, Mozos A, Llobet D, Dolcet X, Pons C, de Herreros AG, Matias-Guiu X, Prat $\mathrm{J}$ : Epithelial to mesenchymal transition in early stage endometrioid endometrial carcinoma. Hum Pathol 2012;43:632-643.

15 Monge M, Doll A, Colas E, Gil-Moreno A, Castellvi J, Garcia A, Colome N, Perez-Benavente A, Pedrola N, Lopez-Lopez R, Dolcet X, Ramon Y Cajal S, Xercavins J, Matias-Guiu X, Canals F, Reventos J, Abal M: Subtractive proteomic approach to the endometrial carcinoma invasion front. J Proteome Res 2009;8: 4676-4684.

16 Jove M, Gatius S, Yeramian A, Portero-Otin M, Eritja N, Santacana M, Colas E, Ruiz M, Pamplona R, Matias-Guiu X: Metabotyping human endometrioid endometrial adenocarcinoma reveals an implication of endocannabinoid metabolism. Oncotarget 2016;7: 52364-52374.

17 Fusté N, Castelblanco E, Felip I, Santacana M, Fernández-Hernández R, Gatius S, Pedraza N, Cemeli T, Pallares J, Valls J, Tarres M, Ferrezuelo F, Dolcet X, Matias-Guiu X, Garí E: Characterization of cytoplasmic cyclin D1 as a marker of invasiveness in cancer. Oncotarget 2016;7:26979-26991.

18 Espinosa I, Catasus L, D Angelo E, Mozos A, Pedrola N, Bértolo C, Ferrer I, Zannoni GF, West RB, van de Rijn M, Matias-Guiu X, Prat J: Stromal signatures in endometrioid endometrial carcinomas. Mod Pathol 2014;27: 631-639.

19 Mota A, Colas E, Sanz PG, Campoy I, Rojo A, Gatius S, Garcia A, Chiva A, Alonso MS, Gil A, Gonzalez-Tallada X, Diaz B, Vidal A, Ziober P, Bobinski, MS, Lopez R, Abal M, Reventos J, Matias-Guiu X, Moreno-Bueno $\mathrm{G}$ : Genetic analysis of uterine aspirates improves the diagnostic value and captures the intra-tumor heterogeneity of endometrial cancers. Mod Pathol 2017;30:134-145. 